\section{Cancer and schizophrenia}

The negative finding of the recent paper by Goldacre et al (2005) is an important addition to studies attempting to confirm or disprove the 'epidemiologic puzzle' (Jablensky \& Lawrence, 2001), but the evidence remains ambiguous regarding the overall risk of cancer in people with schizophrenia. In three of five comparisons with reference populations conducted between 1992 and 2003 (see Grinshpoon et al, 2005), males with schizophrenia had a reduced risk of cancer. No reduction was found among females in four comparisons but decreased risk was reported in one of two comparisons of both males and females. Two recent studies (Dalton et al, 2005; Grinshpoon et al, 2005) mostly found reduced risk. When evaluating these results, it is important to recall that people with schizophrenia face many health and service hazards that may increase their risk for cancer (Grinshpoon et al, 2005). We therefore suggest that results in this area should not be stopped prematurely, especially since one study (Lichtermann et al, 2001), but not another (Dalton et al, 2004), found a reduced risk of cancer among first-degree relatives of patients with schizophrenia, an indication of a genetic factor (Park et al, 2004).

The study by Goldacre et al (2005) had some limitations, as acknowledged by the authors. We wonder whether a diagnosis of schizophrenia at the time of the first admission may not constitute an additional limitation. Patients admitted with an early diagnosis of schizophrenia but who later received other psychiatric diagnoses might have diluted the risk (Carney et al, 2004), whereas others who did not have a diagnosis of schizophrenia on first admission but did on later contact might have been lost to the enquiry. We also wonder whether the decision to exclude some people from the reference population for selected cancers was sound. Admittedly, dietary factors may be imputed for those conditions selected for elimination as well as for cancer risk. We look forward to a repetition of the analysis after their inclusion.

Carney, C. P., Woolson, R. F., Jones, L., et al (2004) Occurrence of cancer among people with mental health claims in an insured population. Psychosomatic Medicine, 66, 735-743.

Dalton, S. O., Laursen, T. M., Mellemkjaer, L., et al (2004) Risk for cancer in parents of patients with schizophrenia. American Journal of Psychiatry, 161, 903-908.
Dalton, S. O., Mellemkjaer, L., Thomassen, L., et al (2005) Risk for cancer in a cohort of patients hospitalized for schizophrenia in Denmark, 1969-1993. Schizophrenia Research, 75, 315-324.

Goldacre, M. J., Kurina, L. M., Wotton, C. J., et al (2005) Schizophrenia and cancer: an epidemiological study. British Journal of Psychiatry, 187, 334-338.

Grinshpoon, A., Barchana, M., Ponizovsky, A., et al (2005) Cancer in schizophrenia: is the risk higher or lower? Schizophrenia Research, 73, 333-34l.

Jablensky, A., Lawrence, D. (200I) Schizophrenia and cancer: is there a need to invoke a protective gene? Archives of General Psychiatry, 58, 579-580.

Lichtermann, D., Ekelund, J., Pukkala, E., et al (200I) Incidence of cancer among persons with schizophrenia and their relatives. Archives of General Psychiatry, $\mathbf{5 8}$, 573-578.

Park, J. K., Lee, H. J., Kim, J.W., et al (2004)

Differences in p53 gene polymorphisms between Korean schizophrenia and lung cancer patients. Schizophrenia Research, 67, 71-74.

I. Levav Mental Health Services, Ministry of Health, 2 Ben Tabai Street, Jerusalem 9359I, Israel. E-mail: levavm@zahav.net.il

A. Ponizovsky, A. Grinshpoon Mental Health Services, Ministry of Health, Jerusalem, Israel

Authors' reply: We agree that further evidence is needed to gain greater certainty about whether or not cancer risk is altered in people with schizophrenia. By their nature, observational epidemiological studies include more biases and confounding than randomised controlled trials, but the latter are not an option for studying this association.

We included people in the schizophrenia cohort if they had a discharge diagnosis of schizophrenia at any admission and not just at the first admission. We accept that there could be a dilution effect from early misclassification, but it seems unlikely that this would completely reverse any real and substantial inverse association between schizophrenia and cancer.

We excluded people with appendicectomy, haemorrhoids and inguinal hernia from the reference cohort when studying colorectal cancer because we knew, from other work, that they have a significantly increased risk (albeit fairly small). We therefore felt that, in principle, they were inappropriate for the colorectal cancer analyses. However, this was more a decision on principle than one with much practical effect. Comparing the schizophrenia cohort with the reference cohort, including all people in the reference cohort, the rate ratio for cancer of the rectum fell to 0.55 (95\% CI 0.31-0.90), compared with
$0.57(0.33-0.93)$ reported by us. The rate ratio for cancer of the colon, including all the reference cohort, fell to 0.59 (0.39-0.85) compared with 0.72 (0.50-1.01) reported by us. Thus, a result on the borderline of significance became significant; but we consider that it was right to exclude the three reference groups as in the original analysis. None the less, the case does seem to be building, considering results from other studies as well as ours, that there may be a deficit of colorectal cancer in people with schizophrenia. As suggested by Dr Levav and his colleagues, it is unclear whether this is a result of confounding with dietary factors. Finally, we would like to correct a typographical error in the footnote to our table: 'superficial injury and confusion' should have read 'superficial injury and contusion'!

M. J. Goldacre Unit of Health-Care Epidemiology, Department of Public Health, University of Oxford, Old Road Campus, Old Road, Oxford OX3 7LF, UK.

E-mail: michael.goldacre@dphpc.ox.ac.uk

L. M. Kurina, C. J.Wotton, D. Yeates,

V. Seagroatt Unit of Health-Care Epidemiology, Department of Public Health, University of Oxford, Oxford, UK

\section{Schizophrenia, weight gain and atypical antipsychotics}

Thakore (2005) highlights the increased prevalence of the metabolic syndrome in schizophrenia. He briefly discusses the relationship between atypical antipsychotic drugs, weight gain and abnormal glucose and lipid metabolism. He rightly concludes that this relationship is poorly understood and that much of the evidence is contradictory or of dubious quality. Unfortunately this narrow focus on the aetiology of the metabolic syndrome risks diverting attention from the urgent need to reduce obesity among people with schizophrenia.

There is good evidence that people with schizophrenia have a high and growing cardiovascular mortality (Ösby et al, 2000). Many also have multiple lifestylerelated cardiovascular risk factors such as smoking, lack of exercise and poor diet (Brown et al, 1999), none of which are convincingly susceptible to modification. Schizophrenia may also be associated with intrinsic metabolic disadvantage (Thakore, 2005). 
The data on atypical antipsychotic drugs, weight gain and metabolic dysregulation come from a heterogeneous collection of largely uncontrolled studies, but there is no doubt that these drugs induce weight gain and that some are worse than others. 'First do no harm'. There can be no justification for continuing to prescribe particular atypical antipsychotic drugs which cause serious weight gain to a population who are already at high risk of cardiovascular disease. Equally effective alternatives are readily available and are no more expensive. Obesity increases cardiovascular mortality by $50 \%$ (McGee, 2005). We must stop regarding weight gain as an acceptable price to pay for control of psychiatric symptoms.

\section{Declaration of interest}

S.B. has attended many educational functions supported by pharmaceutical companies but has no other links with the pharmaceutical industry.

Brown, S., Birtwistle, J., Roe, L., et al (1999) The unhealthy lifestyle of people with schizophrenia. Psychological Medicine, 29, 697-701.

McGee, D. L. (2005) Body mass index and mortality: meta-analysis based on person-level data from twentysix observational studies. Annals of Epidemiology, 15 87-97.

Ösby, U., Correia, N., Brandt, L., et al (2000) Time trends in schizophrenia mortality in Stockholm County, Sweden: cohort study. BMJ, 32I, 483-484.

Thakore, J. H. (2005) Metabolic syndrome and schizophrenia. British Journal of Psychiatry, 186, 455-456.

S. Brown Canon House, 6 Canon Street, Shirley, Southampton SOI5 5PQ, UK.

E-mail: Steve.Brown@wht.nhs.uk

\section{Akathisia as a risk factor for suicide}

Hawton et al (2005) have produced a comprehensive, systematic review of risk factors for suicide in schizophrenia. The study questions the fundamental procedures that are an integral part of our clinical assessment of this vulnerable group of patients. Suicide is notoriously difficult to predict because of the rarity of the event, the obvious ethical problems of designing informative studies and the uncertainty about risk factors. However, although there is no study of akathisia and suicide that fulfils their strict inclusion criteria, there is more research available than the case reports mentioned (for example, Chow et al, 1997; Hansen, 2001; Hansen et al, 2004). We found no association between akathisia and suicidality in a group of 90 patients with treatment-resistant schizophrenia (Hansen et al, 2004). Akathisia may, however, have a very different impact on patients at different stages of their illness and according to the duration of treatment. Akathisia emerging early in treatment or after increases in dosages may be the more malignant in terms of distress.

Hawton et al also identified agitation (motor restlessness), impulsivity and depression as risk factors but not akathisia. However, akathisia could contribute to or be confused with any of these three identified risk factors.

There is also evidence that akathisia can occur as a consequence of antidepressant treatment, which is common in patients with schizophrenia (Muller-Oerlinghausen \& Berghofer, 1999; Hansen \& Wilkinson, 2001). Whether there is an additive effect of antipsychotic and antidepressant medication on the intensity and duration of akathisia is not yet known. None the less, in our opinion, it would be premature to exclude akathisia from a role in the complex web of factors that lead to suicide in schizophrenia and perhaps also in other conditions.

Chow, L. Y., Chung, D., Leung, V., et al (1997) Suicide attempt due to metoclopramide-induced akathisia. International Journal of Clinical Practice, 5I, 330-33I.

Hansen, L. (200I) A critical review of akathisia - and its possible association with suicidal behaviour. Human Psychopharmacology, 16, 495-505.

Hansen, L. \& Wilkinson, D. (200I) Drug induced akathisia, suicidal ideation and its treatment in the elderly. International Journal of Geriatric Psychiatry, $\mathbf{9}$ $231-233$

Hansen, L., Jones, R. \& Kingdon, D. (2004) No association between akathisia or parkinsonism and suicidality in treatment resistant schizophrenia. Journal of Psychopharmacology, 18, 384-387.

Hawton, K., Sutton, L., Haw, C., et al (2005)

Schizophrenia and suicide: systematic review of risk factors. British Journal of Psychiatry, 187, 9-20.

Muller-Oerlinghausen, B. \& Berghofer, A. (1999) Antidepressants and suicidal risk. Journal of Clinical Psychiatry, 60, 94-99.

L. Hansen Department of Psychiatry, Royal South Hants Hospital, Brintons Terrace, Southampton SOI4 OYG, UK.

E-mail: Ih4@soton.ac.uk

D. Kingdom Department of Psychiatry, Royal South Hants Hospital, Southampton, UK

\section{Brief psychotherapy for Alzheimer's disease}

I read with interest the paper by Burns et al (2005). This study into an under-researched and important matter is welcome. However, I would like to comment on the conclusions.

The authors quite appropriately comment that the lack of any quantifiable effect of their psychotherapy could result from the small sample size or the relative insensitivity of the outcome measures. They present qualitative data on participants' experience of the psychotherapy which show the therapy in a positive light. The collection of these data was highly biased, since participants in the 'standard care' arm of the trial were not asked about their experience of their treatment. In addition, these patients were not followed-up in the same way as those receiving the therapy. I suspect that if multidisciplinary, holistic care were being provided as it should, these patients would have made equally positive comments about their community psychiatric nurse, social worker, psychiatrist or general practitioner.

The authors of this study have neither devised the adapted therapy (this was described by Brierley et al, 2003), nor have they shown that the therapy works. Hence I disagree with the authors' main conclusion that 'this study shows it is possible to adapt a model of psychotherapy for those with Alzheimer's disease'. They have none the less presented some interesting preliminary data, suggesting a potential benefit of the therapy. I look forward to further research in this area.

Brierley, E., Guthrie, E., Busby, C., et al (2003) Psychodynamic interpersonal therapy for early Alzheimer's disease. British Journal of Psychotherapy, 19 435-446.

Burns, A., Guthrie, E., Marino-Francis, F., et al (2005) Brief psychotherapy in Alzheimer's disease. Randomised controlled trial. British Journal of Psychiatry, 187, 143-147.

D. White Edward Street Hospital, West Bromwich, West Midlands B70 8NL, UK. E-mail: DavidWhite@smhsct.nhs.uk

Author's reply: Dr White has raised some important points. The qualitative data on the participants' experience was only a tiny part of the study and, although agreeing with the points made, I feel they are hardly relevant to the main thrust of the work. Dr White is correct that we did not devise 